\title{
The Optimized White Differential Equation Based on the Original Grey Differential Equation
}

\author{
ZHOU Rui ${ }^{\mathrm{a},{ }^{* 1}}$, LI Ren-guo ${ }^{\mathrm{a},{ }^{* 2}}$, CHEN Yao ${ }^{\mathrm{a}, * 3}$ \\ ${ }^{a}$ Department of Mathematics and Information, China West Normal University, Nanchong, Sichuan, China
}

\begin{abstract}
This paper starting from the original grey differential equations, through finding the relationship between the raw data $x^{(0)}(k)$ and the derivative of its $1-A G O$, constructed a new white differential equation which equal to the original grey differential equation, at the same time, getting the new $\operatorname{GM}(1,1)$ model which closer to the changes of data. Through the modeling and prediction of the standard index series, this model not only adapts to low growth index series, but also adapts to high-growth index series, and the simulation accuracy and prediction accuracy are high.
\end{abstract}

Index Terms: GM (1,1); Original Grey Differential Equation; Equivalent; White Differential Equation; Optimization

(C) 2011 Published by MECS Publisher. Selection and/or peer review under responsibility of the International Conference on E-Business System and Education Technology

\section{Introduction}

Since Mr Deng Ju-long found the grey system theory in the eighties of last century, after almost three decades of development, the theory has been widely used in various areas of national product ${ }^{[1]}$. As the important part of the grey system theory, the $\operatorname{GM}(1,1)$ model has become the research focus, many scholars have further investigated in improving the model's precision and widening the model's applicable scope ${ }^{[3-10]}$. But when the white differential equation founded, Mr Deng Ju-long made that:" $\operatorname{GM}(1,1)$ white model itself and all results derived out from the white model just establish only when they are not contradictory with the defining type, otherwise invalid" "[2]. Based on this idea, this paper through finding the relationship of the raw data $x^{(0)}(k)$ and the derivative of its $1-A G O$, constructed a new kind white differential equation which equaled with the original grey differential equation, at the same time, getting the new $\operatorname{GM}(1,1)$ model which closer to the changes of data. 


\section{The Optimization of GM(1,1) Model}

Let $X^{(0)}=\left\{x^{(0)}(1), x^{(0)}(2), \cdots x^{(0)}(n)\right\}$ is the original series, and the $1-A G O$ series of $X^{(0)}$ is $X^{(1)}=\left\{x^{(1)}(1), x^{(1)}(2), \cdots x^{(1)}(n)\right\}$, among them $x^{(1)}(k)=\sum_{i=1}^{k} x^{(0)}(i)$

Theorem 1 When $x^{(1)}(t)=C e^{A t}+D$ (among them A、C、D are all the constants), the original grey differential equation $x^{(0)}+a x^{(1)}=b$ and the white differential equation $\frac{\left(e^{A}-1\right)}{A e^{A}} \cdot \frac{d x^{(1)}}{d t}+a x^{(1)}=b$ equivalent.

Proof: Because $x^{(1)}(t)=C e^{A t}+D$ (among them A、C、D are all the constants), according with the relevant definitions we can get,

$$
\begin{aligned}
& x^{(0)}(t)=x^{(1)}(t)-x^{(1)}(t-1)=C e^{A(t-1)}\left(e^{A}-1\right) \\
& \left(x^{(1)}(t)\right)^{\prime}=\frac{d x^{(1)}}{d t}=A C e^{A t}=C e^{A(t-1)} \cdot A e^{A}
\end{aligned}
$$

Comparing (1) and (2) can get

$$
x^{(0)}(k)=\left.\frac{\left(e^{A}-1\right)}{A e^{A}} \cdot \frac{d x^{(1)}}{d t}\right|_{t=k}
$$

And wether get which values, this equality always set up.

Put (3) into $x^{(0)}+a x^{(1)}=b$, getting :

$$
\frac{\left(e^{A}-1\right)}{A e^{A}} \cdot \frac{d x^{(1)}}{d t}+a x^{(1)}=b
$$

And when $x^{(1)}(t)=C e^{A t}+D$, (4) is the equally white differential equation with grey equation differential.

End

So based on the original grey differential equation $x^{(0)}+a x^{(1)}=b$, getting the optimized GM $(1,1)$ model as follow: 
Theorem 2 Let $x^{(0)}=\left\{x^{(0)}(1), x^{(0)}(2), \cdots, x^{(0)}(n)\right\}$ be nonnegative pre-smooth series, $x^{(1)}$ is $1-A G O_{\text {series of }} x^{(0)}$, and $x^{(1)}(t)=B e^{A t}+C_{\text {(among them A } 、 \mathrm{C} 、 \mathrm{D} \text { are all the constants). If }}$

$\hat{a}=(a, b)^{T}$ be the parameter of the equation, and $Y=\left(\begin{array}{c}x^{(0)}(2) \\ x^{(0)}(3) \\ \vdots \\ x^{(0)}(n)\end{array}\right), \quad B=\left(\begin{array}{cc}-x^{(1)}(2) & 1 \\ -x^{(1)}(3) & 1 \\ \vdots & \vdots \\ -x^{(1)}(n) & 1\end{array}\right)$, then the parameters of the least square estimate in the grey differential equation $\hat{a}=(a, b)^{T}=\left(B^{T} B\right)^{-1} B^{T} Y$ then

(1) The new continuous solution of the white equation is

$$
x^{(1)}(t)=\left[x^{(0)}(1)-\frac{b}{a}\right] e^{\frac{-a \cdot(t-1) \cdot A \cdot e^{A}}{e^{A}-1}}+\frac{b}{a}
$$

(2) The new discrete solution of the white equation is

$$
x^{(1)}(k)=\left[x^{(0)}(1)-\frac{b}{a}\right] e^{\frac{-a \cdot(k-1) \cdot A \cdot e^{A}}{e^{A}-1}}+\frac{b}{a}, k=2,3, \cdots
$$

(3) The restore value is $\hat{x}^{(0)}(k+1)=\hat{x}^{(1)}(k+1)-\hat{x}^{(1)}(k), k=2,3, \cdots$;

\section{The Process of Modeling Optimized GM(1,1) Model}

The precondition of establishing optimized GM $(1,1)$ model is $x^{(1)}(t)=C e^{A t}+D$ (among them A, C ,D are undetermined constants )that means the original data must be expressed by $x^{(0)}(k)=P e^{A(k-1)} \quad$ among them $\mathrm{P}$ is a constant ). Under this precondition, $e^{A}=\frac{x^{(0)}(k)}{x^{(0)}(k-1)}$ is always a constant. But in the practical constant. So the new model is not practical. To this problem, the model must be optimized again.

$$
e^{A_{i}}=\frac{x^{(0)}(i)}{x^{(0)}(i-1)} \text { is not a }
$$

If the original series is $X^{(0)}=\left\{x^{(0)}(1), x^{(0)}(2), \cdots x^{(0)}(n)\right\}$, then $1-A G O$ series of $X^{(0)}$ is $X^{(1)}=\left\{x^{(1)}(1), x^{(1)}(2), \cdots x^{(1)}(n)\right\}, x^{(1)}(k)=\sum_{i=1}^{k} x^{(0)}(i)$.

Let $\quad=\frac{x^{(0)}(i)}{x^{(0)}(i-1)}, \mathrm{i}=2,3 \ldots \mathrm{n}$, then

$$
A_{i}=\ln \left(\frac{x^{(0)}(i)}{x^{(0)}(i-1)}\right)
$$

$\mathrm{i}=2,3 \ldots \mathrm{n}$. And $e^{A_{i}}, A_{i}$ replace the $e^{A}$ and $A$ which in theorem 2 .

Now getting the new optimized GM $(1,1)$ as follow: 
Theorem 3 Let $x^{(0)}=\left\{x^{(0)}(1), x^{(0)}(2), \cdots, x^{(0)}(n)\right\}$ be nonnegative pre-smooth series, $x^{(1)}$ be the

$$
1-A G O \text { series of } x^{(0)} \text {. If } \hat{a}_{=}(a, b)^{T} \text { the parameter of the equation, and } \quad Y=\left(\begin{array}{c}
x^{(0)}(2) \\
x^{(0)}(3) \\
\vdots \\
x^{(0)}(n)
\end{array}\right) \text {, }
$$

$$
B=\left(\begin{array}{cc}
-x^{(1)}(2) & 1 \\
-x^{(1)}(3) & 1 \\
\vdots & \vdots \\
-x^{(1)}(n) & 1
\end{array}\right)
$$

$\hat{a}=(a, b)^{T}=\left(B^{T} B\right)^{-1} B^{T} Y$,then

(1) The new continuous solution of the white equation is

$$
x^{(1)}(t)=\left[x^{(0)}(1)-\frac{b}{a}\right] e^{\frac{-a \cdot(t-1) \cdot x^{(0)}(t) \cdot \ln \left(\frac{x^{(0)}(t)}{x^{(0)}(t-1)}\right)}{x^{(0)}(t)-x^{(0)}(t-1)}}+\frac{b}{a} \text {; }
$$

(2) The new discrete solution of the white equation is

$$
x^{(1)}(k)=\left[x^{(0)}(1)-\frac{b}{a}\right] e^{\frac{-a \cdot(t-1) \cdot x^{(0)}(k) \cdot \ln \left(\frac{x^{(0)}(k)}{x^{(0)}(k-1)}\right)}{x^{(0)}(k)-x^{(0)}(k-1)}}+\frac{b}{a},
$$
$k=2,3, \cdots \mathrm{n}$;

(3) According to "The principle of new information priority" getting the prediction equation: $x^{(1)}(k+1)=\left[x^{(0)}(1)-\frac{b}{a}\right] e^{\frac{-a \cdot(t-1) \cdot x^{(0)}(k) \cdot \ln \left(\frac{x^{(0)}(n)}{x^{(0)}(n-1)}\right)}{x^{(0)}(n)-x^{(0)}(n-1)}}+\frac{b}{a}, \quad k=n, n+1 \cdots$.

(4) The restore value is

$$
\hat{x^{(0)}}(k+1)=\hat{x^{(1)}}(k+1)-\hat{x^{(1)}}(k), \quad k=2,3, \cdots \text {; }
$$

\section{Comparison of the Precision of Data Simulation}

Take $x^{(0)}(k+1)=e^{-a k}$ as an example, and then we have $x_{i}^{(0)}=\left\{x_{i}^{(0)}(1), x_{i}^{(0)}(2), \cdots x_{i}^{(0)}(n)\right\}, k=1,2,3 \cdots 6$. And use the original GM $(1,1)$ model as M1, model of reference [2] as M2and model of this paper as M3 to predict. 
Table 1. The Original Series

\begin{tabular}{llllllll}
\hline$-a$ & $i$ & $x_{i}^{(0)}(1)$ & $x_{i}^{(0)}(2)$ & $x_{i}^{(0)}(3)$ & $x_{i}^{(0)}(4)$ & $x_{i}^{(0)}(5)$ & $x_{i}^{(0)}(6)$ \\
\hline 0.1 & 1 & 1.0 & 1.1052 & 1.2214 & 1.3499 & 1.4918 & 1.6487 \\
0.3 & 2 & 1.0 & 1.3499 & 1.8221 & 2.4596 & 3.3201 & 4.4817 \\
0.5 & 3 & 1.0 & 1.6487 & 2.7183 & 4.4817 & 7.389 & 12.1825 \\
0.8 & 4 & 1.0 & 2.2255 & 4.953 & 11.0232 & 24.5325 & 54.5982 \\
1.0 & 5 & 1.0 & 2.7183 & 7.389 & 20.0855 & 54.5982 & 148.4132 \\
1.5 & 6 & 1.0 & 4.4817 & 20.0855 & 90.0171 & 403.4288 & 1808.0424 \\
1.8 & 7 & 1.0 & 6.0496 & 36.5982 & 221.1064 & 1339.4308 & 8103.0839 \\
2.0 & 8 & 1.0 & 7.3891 & 54.5982 & 403.4287 & 2980.9579 & 22026.4657 \\
3.0 & 9 & 1.0 & 20.08 & 403.42 & 8103.08 & 162754.79 & 3269017.37 \\
\hline
\end{tabular}

Table 2. Comparison of the Simulation Precision

\begin{tabular}{|c|c|c|c|c|c|c|c|c|}
\hline$-\mathbf{a}$ & & 1 & 2 & 3 & 4 & 5 & 6 & Average error $\%$ \\
\hline \multirow{3}{*}{0.1} & M1 & 0 & 0.0876625 & 0.0940814 & 0.1066281 & 0.111198 & 0.120834 & 0.104008 \\
\hline & M2 & 0 & 0.4729089 & 0.4072969 & 0.3355724 & 0.271905 & 0.203195 & 0.338175 \\
\hline & M3 & 0 & 0.0016535 & 0.0006224 & 0.0000653 & 0.0012815 & 0.000827 & 0.000741 \\
\hline \multirow{3}{*}{0.3} & M1 & 0 & 0.8623506 & 1.0792191 & 1.300537 & 1.5200909 & 1.740273 & 1.300494 \\
\hline & M2 & 0 & 1.3160696 & 1.2340048 & 1.1469605 & 1.0612932 & 0.974550 & 1.146575 \\
\hline & M3 & 0 & 0.0012404 & 0.0017363 & 0.0008671 & 0.0000458 & 0.000436 & 0.000721 \\
\hline \multirow{3}{*}{0.5} & M1 & 0 & 2.5688891 & 3.5558678 & 4.5305732 & 5.494881 & 6.451143 & 4.520271 \\
\hline & M2 & 0 & 2.0655219 & 2.0094938 & 1.9559263 & 1.9029764 & 1.848208 & 1.956425 \\
\hline & M3 & 0 & 0.0001108 & 0.0005422 & 0.001017 & 0.0007982 & 0.000928 & 0.000566 \\
\hline \multirow{3}{*}{0.8} & M1 & 0 & 7.1362838 & 10.787576 & 14.295032 & 17.663597 & 20.90019 & 14.15653 \\
\hline & M2 & 0 & 3.0625163 & 3.0445781 & 3.0269682 & 3.0106039 & 2.993673 & 3.027668 \\
\hline & M3 & 0 & 0.0009656 & 0.002194 & 0.001413 & 0.0001291 & 0.001006 & 0.000951 \\
\hline \multirow{3}{*}{1.0} & M1 & 0 & 11.543467 & 17.996636 & 23.980549 & 29.527595 & 34.66966 & 23.54358 \\
\hline & M2 & 0 & 3.645538 & 3.6405011 & 3.6333885 & 3.6265874 & 3.620138 & 3.633230 \\
\hline & M3 & 0 & 0.0000603 & 0.0004651 & 0.001391 & 0.0008119 & 0.000229 & 0.000493 \\
\hline \multirow{3}{*}{1.5} & M1 & 0 & 26.417176 & 41.518302 & 53.520549 & 63.059523 & 70.64080 & 51.03127 \\
\hline & M2 & 0 & 4.7467368 & 4.7467189 & 4.7460967 & 4.7455926 & 4.745129 & 4.746054 \\
\hline & M3 & 0 & 0.0006301 & 0.0003254 & 0.001015 & 0.0007916 & 0.000700 & 0.000577 \\
\hline \multirow{3}{*}{1.8} & M1 & 0 & 36.832816 & 56.255155 & 69.705425 & 79.020111 & 85.47080 & 65.45686 \\
\hline & M2 & 0 & 5.1338165 & 5.1330021 & 5.1328225 & 5.1327235 & 5.132638 & 5.133000 \\
\hline & M3 & 0 & 0.004412 & 0.005303 & 0.004418 & 0.004257 & 0.004223 & 0.003769 \\
\hline \multirow{3}{*}{2.0} & M1 & 0 & 43.862241 & 65.151782 & 78.367616 & 86.571496 & 91.66413 & 73.12345 \\
\hline & M2 & 0 & 5.2674478 & 5.2679511 & 5.268046 & 5.2679988 & 5.267970 & 5.267882 \\
\hline & M3 & 0 & 0.003801 & 0.004514 & 0.003903 & 0.003615 & 0.00366 & 0.003249 \\
\hline \multirow{3}{*}{3.0} & M1 & 0 & 73.21316 & 91.84847 & 97.51940 & 99.24512 & 99.770 & 76.933 \\
\hline & M2 & 0 & 5.08825 & 5.0881 & 5.0881 & 5.0881 & 5.0881 & 5.08813 \\
\hline & M3 & 0 & 0.0028098 & 0.0031082 & 0.002784 & 0.002790 & 0.00278 & 0.002380 \\
\hline
\end{tabular}

From Table 2 and Table 3, when the original series is a low growth series, the simulation accuracy and prediction accuracy of all models are high, but as the development coefficient become larger, the he simulation 
accuracy and prediction accuracy of original model become lower. Especially, when $|a|>2$, the error is $99 \%$ ! Although the simulation accuracy and prediction accuracy of M2 is higher than M1, the overall effect is not very ideal. But for the model of this paper, even if $|a|>2$, the simulation accuracy and prediction accuracy are all higher than $99.99 \%$, the effect is good.

Table 3. Comparison of the Forecasting Precision (Relative error \%)

\begin{tabular}{lllllll}
\hline & $\boldsymbol{a}$ & $\mathbf{0 . 1}$ & $\mathbf{0 . 3}$ & $\mathbf{0 . 5}$ & $\mathbf{0 . 8}$ & $\mathbf{1 . 0}$ \\
\hline M1 & 1step error & 0.1289 & 1.9604 & 7.3970 & 24.0093 & 39.4369 \\
$\mathbf{M 2}$ & $\mathbf{1 s t e p}$ error & 0.1333 & 0.8890 & 1.7940 & 2.9772 & 3.6135 \\
$\mathbf{M 3}$ & $\mathbf{1 s t e p}$ error & 0.00135 & 0.00071 & 0.00121 & 0.00126 & 0.00025 \\
\hline M1 & 2step error & 0.1367 & 2.1791 & 8.3332 & 26.9963 & 43.8559 \\
M2 & 2step error & 0.0650 & 0.8032 & 1.7400 & 2.9606 & 3.6070 \\
M3 & $\mathbf{2 s t e p ~ e r r o r ~}$ & 0.00225 & 0.000746 & 0.00144 & 0.00143 & 0.00026 \\
\hline M1 & $\mathbf{5}$ step error & 0.1601 & 2.8322 & 11.0855 & 35.2711 & 55.2708 \\
M2 & $\mathbf{5}$ step error & 0.1394 & 0.5462 & 1.5784 & 2.9105 & 3.5874 \\
M3 & $\mathbf{5}$ step error & 0.00493 & 0.000846 & 0.00214 & 0.00194 & 0.00027 \\
\hline M1 & $\mathbf{1 0}$ step error & 0.1991 & 3.9110 & 15.4903 & 47.0312 & 69.3755 \\
M2 & $\mathbf{1 0}$ step error & 0.2808 & 0.1193 & 1.3096 & 2.8272 & 3.5548 \\
M3 & $\mathbf{1 0}$ step error & 0.00943 & 0.001013 & 0.00331 & 0.00278 & 0.00028 \\
\hline
\end{tabular}

\begin{tabular}{llllll}
\hline & $\boldsymbol{- a}$ & $\mathbf{1 . 5}$ & $\mathbf{1 . 8}$ & $\mathbf{2 . 0}$ & $\mathbf{3 . 0}$ \\
\hline M1 & 1step error & 76.6670 & 89.9372 & 94.8254 & 99.9300 \\
M2 & 1step error & 4.7213 & 5.1325 & 5.2679 & 5.0880 \\
M3 & 1step error & 0.0007017 & 0.003937 & 0.003664 & 0.0027891 \\
\hline M1 & 2step error & 81.4556 & 93.0312 & 96.7878 & 99.9787 \\
M2 & 2step error & 4.7171 & 5.1324 & 5.2679 & 5.0880 \\
M3 & 2step error & 0.0007033 & 03003938 & 0.003661 & 0.0027891 \\
\hline M1 & $\mathbf{5}$ step error & 90.6903 & 97.6854 & 99.2316 & 99.9994 \\
M2 & $\mathbf{5}$ step error & 4.7045 & 5.1320 & 5.2678 & 5.0880 \\
M3 & $\mathbf{5}$ step error & 0.0007082 & 0.003941 & 0.003653 & 0.0027891 \\
\hline M1 & $\mathbf{1 0}$ step error & 97.0478 & 99.6313 & 99.9291 & 99.9999 \\
M2 & $\mathbf{1 0}$ step error & 4.6836 & 5.1315 & 5.2677 & 5.0880 \\
M3 & $\mathbf{1 0}$ step error & 0.0007164 & 0.003946 & 0.003640 & 0.0027892 \\
\hline
\end{tabular}

\section{Conclusions}

In this paper, starting from the original grey differential equations, through finding the relationship of the raw data $x^{(0)}(k)$ and the derivative of its $1-A G O$, constructed a new kind white differential equation which equal with the original grey differential equation, at the same time, getting the new $\operatorname{GM}(1,1)$ model which closer to the changes of data. Through the modeling and prediction of the standard index series, this 
model not only adapts to low growth index series, but also adapts to high-growth index series, and the simulation accuracy and prediction accuracy are almost $100 \%$. At the same time, the models remains simple calculation steps of the original GM $(1,1)$ model, and broaden the scope of application of the model.

\section{References}

[1] Liu Si-feng, Dang Yao-guo, Fang Zhi-geng(2004). The Grey System Theory and Its Application [M]. Science Press. Beijing.

[2] Deng Ju-long (2002). Estimate and Decision of Grey System. Huazhong University of Science\& Technology Press, Wuhan.

[3] Luo Dang, Liu Sifeng, Dang Yaoguo(2003). The Optimization of Grey Model GM(1,1).Engineering Science, 8, 50 53

[4] Wang Hong. Optimized GM(1,1)model and Its Prediction Accuracy Analysis[J]. Statistics and Decision 2008,23:36-38.

[5] Dong Fen-yi, TIAN Jun.Optimization integrated background value with original condition for GM( 1,1$)[J]$. Systems Engineering and Electronics.2007. Vol.29. No.3 454-456.

[6] Wang Yinao, Liu Kaidi, Li Yingchuan (2001). GM(1,1) modeling method of optimum the whiting values of grey deribative. Systems Engineering-theory \& Practice, 5, 124 128.

[7] Hu Da-hong, Wei Yong. Applicability of Grey model to the monotonic decreasing sequences and the approximate estimate of parameters[J] .Systems Engineering and Electronics. 2008.9:16-20.

[8] Liu Sifeng, Deng Julong (2000). The range suitable for GM $(1,1)$. Systems Engineering-theory \& Practice, $5,121 \sim 124$.

[9] Dang Yao-guo, Liu Si-feng. Liu Bin. The GM Models that $\mathrm{x}^{(1)}(\mathrm{n})$ be Taken as Initial Value[J]. Chinese Journal of Management Science. 2005,2(1):132-235.

[10] Li Junfeng, Dai Wenzhan(2004).A New Approach of Background Value-Building and Its Application Based on Data Interpolation and Newton-Cores Formula. Systems Engineering-theory \& Practice, 10, 122 126. 